\title{
PRACTICAL APPROACH FOR SOLUTION OF THE PROBLEM OF SHIP MANEUVERABILITY IN WAVES
}

\author{
Evgeni Milanov \\ Institute of Metal Science, Equipment and Technologies \\ with Hydro- and Aerodynamics Centre "Acad. Angel Balevski", \\ Bulgarian Academy of Sciences \\ 1, William Froude St., 9003 Varna, Bulgaria, \\ e-mail:e.milanov@bshc.bg
}

\begin{abstract}
The paper aims to assess the capability of open-source software PDFSTRIP to calculate the ship motions and secondorder drift forces in regular waves. To obtain greater representativeness of the results, the calculations are performed for three ships - two container vessels of the first and third generation and supertanker. The numerical results are compared with the results of model tests conducted in hydrodynamic experimental tanks. Based on the calculated drift load, a procedure has been developed for the preliminary assessment of the ship maneuvers in waves. The developed procedures would be useful as a fast and economically beneficial tool for preliminary estimation of vessel steering qualities in real sea conditions.
\end{abstract}

Keywords: ship maneuvering, wave motions, wave loads.

\section{INTRODUCTION}

Over the years the ship maneuvering qualities have been traditionally analyzed, predicted, and normalized for calm and deep water assuming the negligible influence of external sea conditions. However, given the fact that the assumption of negligible external effects such as wind, current, shallow water, and waves is not strict, many studies have focused on the first three factors, because the concept of accounting for them fits into the time-domain studies of ship maneuverability. In terms of the impact of the sea waves, however, taking it into account is much more complicated because in contrast to the maneuvering, in the seakeeping of the ship the studies are related to the 6 DOF ship motions in waves on a straight course or possibly with some drift

DOI: $10.7546 /$ EngSci.LVIII.21.02.01 
angle and what most important - estimation of vessel seakeeping characteristics is made in the frequency domain and can be made through a statistical, probabilistic or analytical approach.

In real navigation conditions the two problems overlap, when performing the maneuver in waves-induced ship motions interacts with the ship maneuvering motion whereby waves may substantially influence the hydrodynamic forces and thereby change the maneuvering behaviour, and vice versa. This gives rise to the need to study the ship maneuverability in waves and leads to the necessity to combine approaches in the two areas under consideration, which is not an easy task. Since the Energy Efficiency Design Index was introduced by International Maritime Organization, serious concerns regarding the maneuverability of ships in waves have been brought to the forefront. As a result, research into the problem has been initiated in several centers, using experimental, numerical, and hybrid approaches such as project SHOPERA [1]. Within the framework of this project for the study of the ship maneuverability in the above conditions, three navigation scenarios are proposed for consideration when the ship maneuvers are carried out at critically low speeds. However, a serious problem arises in determining the forces of wave drift by model experiment or by the methods of viscous numerical hydrodynamics due to the high cost and time required.

In this regard, due to the known applicability of the potential flow theory codes for calculating the ship oscillation [2-3], in this paper, an attempt is made to determine the average drift forces of the second-order using the opensource program PDSTRIP.

\section{PDSTRIP PROGRAM}

PDSTRIP code solves the potential flow problem around the ship hull by calculation of ship seakeeping 2D hydrodynamic characteristics of hull crosssections oscillating in small amplitude waves near to the free water surface.

Thus, the Airy wave theory is applied for the linear boundary value problem.

Since only the linear components of the ship motion and hull pressure are determined in the PDSTRIP program, thus to estimate the time-averaged drift forces in the regular wave the Boese method is applied.

The estimation of the time-averaged wave drift load is performed by the wetted hull pressure integration. The inertial coordinate system $\xi, \eta, \zeta$ is fixed at mid-ship, and moving forward with the average speed velocity gives the formulae of the longitudinal and transverse time-averaged drift forces in 
the PDSTRIP through expressions (1) and (2), see [4]:

$$
\begin{aligned}
\bar{F}_{\xi}= & \frac{1}{2}\left(m w_{e}^{2} \operatorname{Re}\left(\hat{Y}_{2 s} \hat{Y}_{6}^{*}-\hat{Y}_{3 s} \hat{Y}_{5}^{*}\right)+\frac{1}{2} \rho g \sum_{S B, \text { Port }} \int\left|\hat{Y}_{z r}\right|^{2} \frac{d y_{w}^{+}}{d x} d x\right. \\
& \left.+\frac{1}{2} \rho g\left(\hat{Y}_{4}^{2}+\hat{Y}_{5}^{2}\right)\left[A_{x 0}\left(T+z_{x 0}\right)\right]_{\text {transom }}\right) \zeta_{A}^{2}, \\
\hat{F}_{\eta}= & \frac{1}{2}\left(-m w_{e}^{2} \operatorname{Re}\left(\hat{Y}_{1, s} \hat{Y}_{6}^{*}\right)+\rho g \sum_{S B, \text { Port }} \int y_{w}^{+} \operatorname{Re}\left(\hat{Y}_{z r 0} \hat{Y}_{\zeta w y}^{*}\right) d x\right) \zeta_{A}^{2} .
\end{aligned}
$$

The sense of the above-consolidated equation terms is:

- The first expression is related to rigid body harmonic oscillations in the surge, sway, heave, and pitch modes;

- The second expression is due to the relative motions between the ship and water surface;

- The third expression into equation (1) represents the time-averaged correction for the ships with submerged transom pressure fluctuation.

MATLAB auxiliary codes have been developed for processing the PDSTRIP program output data.

\section{SHIPS GEOMETRY DESCRIPTION}

When choosing ship forms used for numerical investigations, additional and practically the basic argument was the availability of verified experimental data for measured wave drift forces, as well as for the characteristics of the ship motions. The International Towing Tank Conference standard models meet in the best way these conditions: the container vessels S175, DTC, and the supertanker KVLCC2 [5]. In Table 1, their main characteristics are listed.

For the generation of hulls mesh mainly 28 sections have been used. The number of offset points must be at least 15 according to the recommendations of the authors of the code. Numerical studies have shown that doubling the number of offset points on half cross-section improves the predictions by about $3 \%$, which number was considered in the calculations. The hulls were meshed up to the calm waterline. For example, through Figs 1 and 2 the "regular" and "doubled" hull grids of DTC underwater body stern and bow parts are illustrated. 
Table 1. Ships main particulars

\begin{tabular}{|c|c|c|c|}
\hline & S175 & DTC & KVLCC2 \\
\hline$L_{p p}[\mathrm{~m}]$ & 175 & 355 & 320 \\
\hline$B[\mathrm{~m}]$ & 25.4 & 51 & 58 \\
\hline$d[\mathrm{~m}]$ & 9.5 & 14.5 & 20.8 \\
\hline$\nabla\left[\mathrm{m}^{\wedge} 3\right]$ & 24800 & 173468 & 320438 \\
\hline$L C G[\% \mathrm{Lpp}] \mathrm{fwd}+$ & -1.43 & -0.96 & 3.48 \\
\hline$r_{x x}[\mathrm{~m}]$ & 10.16 & 20.30 & 23.20 \\
\hline$r_{y y}[\mathrm{~m}]$ & 47.25 & 87.30 & 80 \\
\hline$r_{z z}[\mathrm{~m}]$ & 47.25 & 87.30 & 80 \\
\hline
\end{tabular}

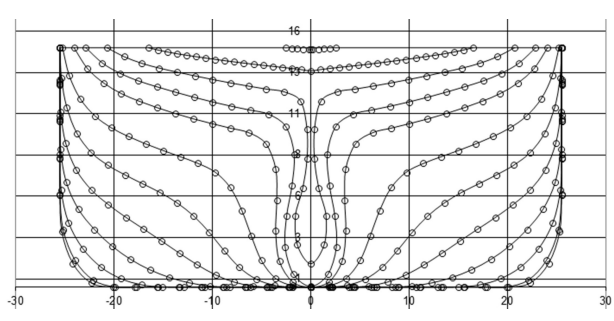

Fig. 1. DTC hull "regular" grid (stern)

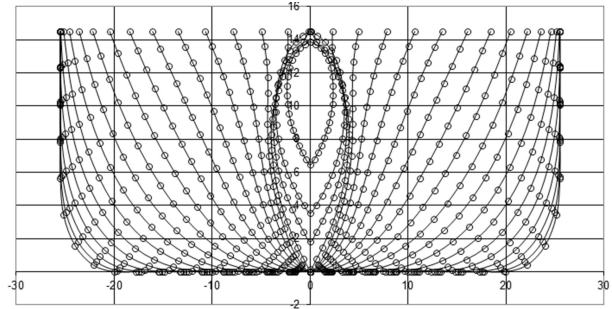

Fig. 2. DTC hull "doubled" grid (bow)

\section{NUMERICAL RESULTS}

In the 3D simulation model for the prediction of the maneuvers of the ship in waves Fig. 3, the time-averaged wave drift forces and moment are included - generalized equations (3)-(5):

$$
\begin{gathered}
m\left(\dot{u}_{0}-v_{0} r_{0}\right)=X_{\text {hull }}+X_{\text {rudder }}+X_{\text {propeller }}+X_{\text {wave }}\{\omega, \mu, U\}, \\
m\left(\dot{v}_{0}-u_{0} r_{0}\right)=Y_{\text {hull }}+Y_{\text {rudder }}+Y_{\text {wave }}\{\omega, \mu, U\}, \\
I_{z z} \dot{r}_{0}=N_{\text {hull }}+N_{\text {rudder }}+N_{\text {wave }}\{\omega, \mu, U\} .
\end{gathered}
$$

Inertial forces are on the left-hand side of the equation, while the equation right-hand side represents hydrodynamic forces and yaw moment, including wave drift load components. The wave forces and yaw moment depend on wave frequency $\omega$; encounter angle $\mu$, and approach speed $U$. The calm water hydrodynamic coefficients are delivered by Planar Motion Mechanism captive model tests.

The above set of ordinary differential equations is solved in time-domain using pre-calculated values of wave load components. These data are stored in the database as well as data for the corresponding Response Amplitude Operator (RAO). The results of these calculations are described below. 


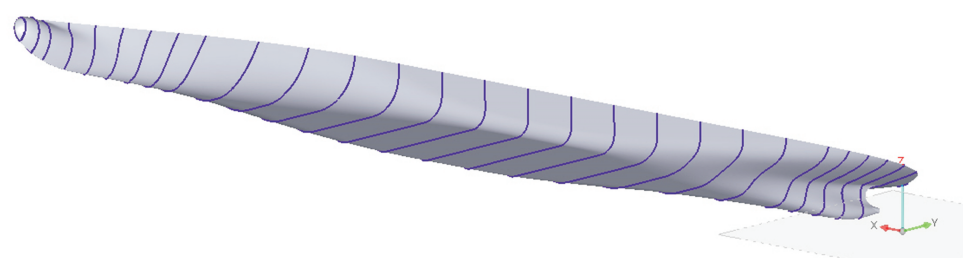

Fig. 3. DTC 3D hull model

\subsection{Motions RAO}

It is known $[3,6]$ that the strip method used in the code calculates the ship motions characteristics with very good accuracy. This is confirmed by exemplary results from the calculations RAO for the heave and pitch motions of the three forms of the hull, given in Figs 4 and 5. The calculated and experimental results [7-9] for the case of head waves (ships DTC and KVLCC2) are in good agreement.

However, the wave encounter angles in the range 0-360 degrees should be considered here. In this regard, the predictions of the responses at an encounter angle of 150 degrees are shown in Fig. 4(a) and Fig. 5(a).

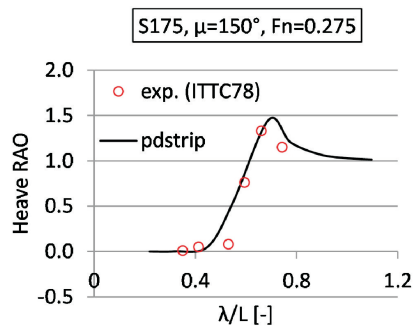

(a)

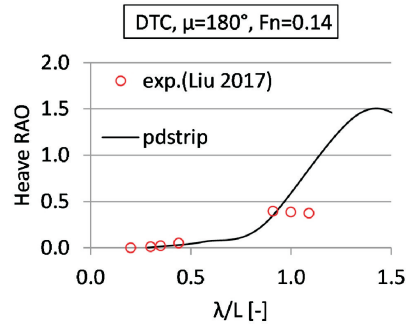

(b)

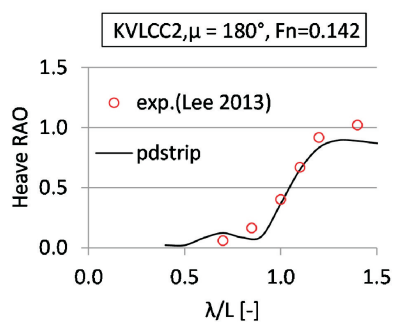

(c)

Fig. 4. Heave Response Amplitude Operators

While the approximation for the vertical motions is good, for the pitch motion for wavelength $\lambda / L>0.7$ the calculated results underestimate the measured ones. Numerical studies performed for other wave directions show satisfactory results suitable for evaluating the considered characteristics. 


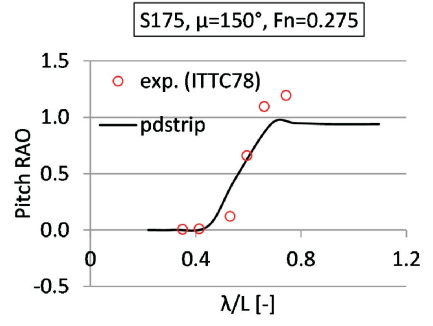

(a)

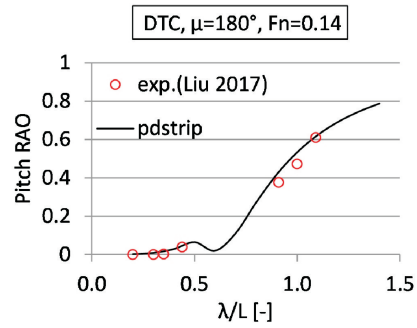

(b)

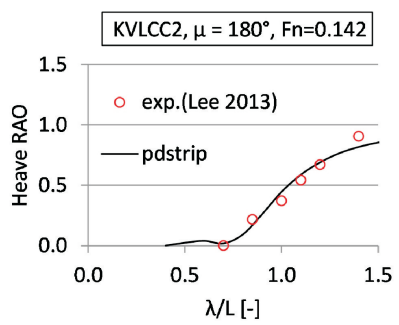

(c)

Fig. 5. Pitch Response Amplitude Operators

\subsection{Second-order wave drift forces and moment}

The drift forces and moments are of fundamental importance in predicting the change in the ship maneuvering qualities in waves. In this paper, we consider the three-dimensional case of the maneuvering motion in regular waves equations (3)-(5). The results of the calculations with PDSTRIP and those of the experiments conducted in a hydrodynamic basin were standardized using the following dimensioning factors:

$$
R_{A W}, Y_{d}=\frac{R_{A W 0}, Y_{d 0}}{\left(\frac{0.5 \rho g \zeta^{2} B^{2}}{L}\right)} ; \quad N_{d}=\frac{N_{d 0}}{\left(0.5 \rho g \zeta^{2} B^{2}\right)}
$$

The values of the additional resistance for ship service speeds calculated using two-dimensional strip methods usually overestimate the value of this quantity [2-3]. This is illustrated, for example, by Fig. 6(a). The experimental data have been published by several authors [10-12]. The reason for this is the two-dimensional nature of the problem formulation. In the program, for the added mass $m_{11}$ the empirical formula is used. On the other hand, at low and zero speeds the approach is good, Fig. 6(b, c). It should be emphasized that the critical scenarios for maneuvering in waves are at low speeds [1]. Therefore, data from the favorable area of $R_{A W}$ estimations will be available.

The results of the calculations of the transverse force and the yaw moment caused by the influence of the regular waves for the three ship hull shapes are shown in Fig. 7. Experimental data are also plotted. It is necessary to indicate the technical difficulties in measuring and extracting this category of forces in the conditions of an experimental basin. For this reason, a significant scatter of the experimental values is observed. In Figure 7 three cases of wave encounter angles are given - 90, 60 and 150 degrees, as the speeds are different, i.e. a 


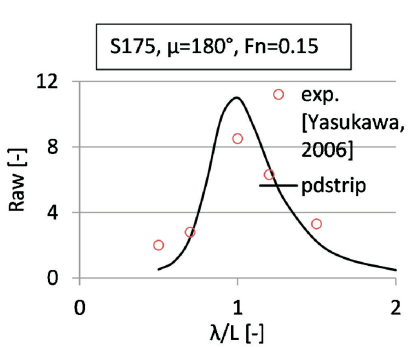

(a)

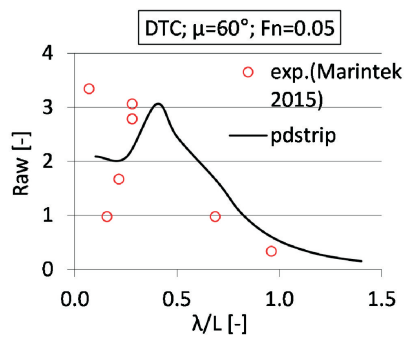

(b)

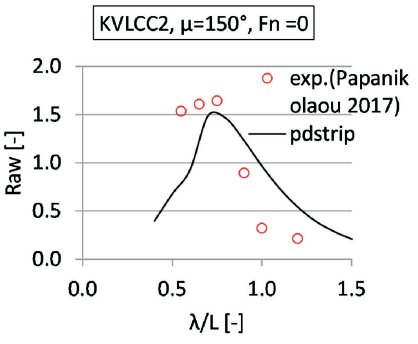

(c)

Fig. 6. Added resistance $R_{A W}$ versus wavelength $\lambda / L$

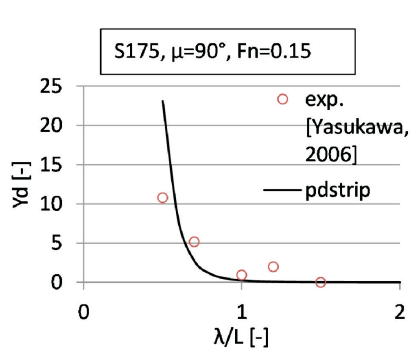

(a)

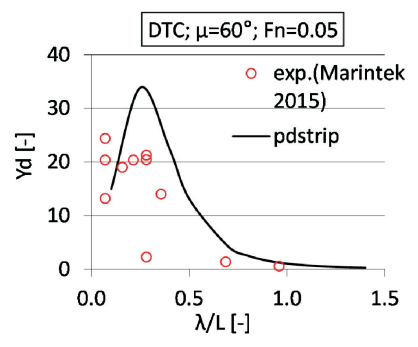

(b)

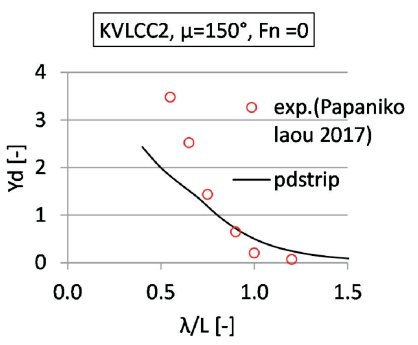

(c)

Fig. 7. Lateral drift force $Y_{d}$ versus wavelength $\lambda / L$

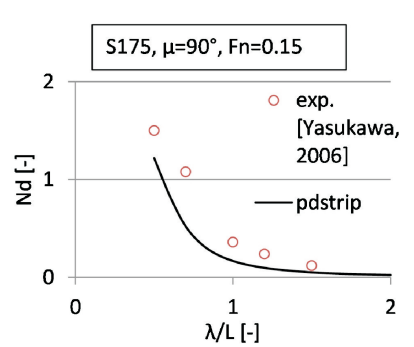

(a)

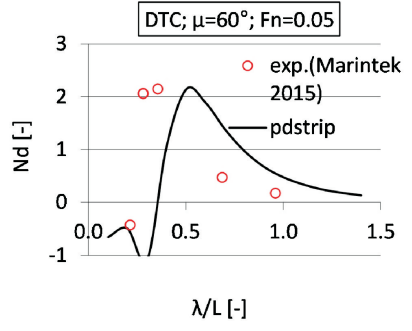

(b)

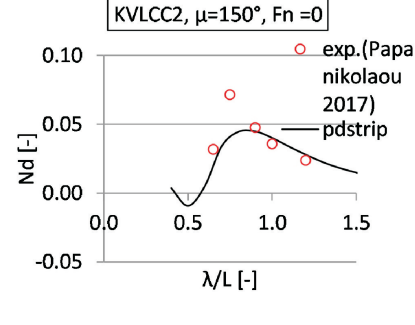

(c)

Fig. 8. Yaw drift moment $N_{d}$ versus wavelength $\lambda / L$ 
range of changes in the parameters of the external environment is covered. Numerical results can be considered good for longer waves about $\lambda / L>0.7$, while for short waves the approximation is evaluative.

For these parameters, the drift moment is also calculated, Fig. 8. In the presence of a scatter of the experimental points, the estimates can be considered good.

\subsection{Database generation}

To solve the equations (3)-(5) of the ship maneuvering in the time domain, it is necessary to calculate wave load values at each step. At this moment to obtain these data by methods of viscous numerical hydrodynamics (CFD) it is time-consuming and costly. The determination of the values under chapters 4.1 and 4.2 utilizing the potential code PDSTRIP is performed in a fast way on a regular PC processor. This inherent quality of the potential codes is the main reason for the present study on the applicability of the obtained results for the wave drift in the model of maneuvering motion.

The procedure consists of the preliminary calculation of added resistance; lateral drift force and yaw drift moment for the set of environmental variables - wave encounter angle, speed, and wavelength. The calculation results are organized in appropriate database, quick access to which is realized with the help of built-in fast MATLAB procedures. This also allows real-time simulation of ship maneuvers in regular waves.

The KVLCC2 supertanker was chosen as an example in the database generation. The matrix of the calculation parameters is given in Table 2. The initial parameters of each simulation case are - frequency of the regular wave, wave steepness, course, speed. Under these conditions, for 13 frequencies in the database, the arrays $\{9 \times 11\}$ for the wave drift forces and moment are available. Studies have shown that a linear approximation is sufficient when determining intermediate values due to the density of points in the preliminary calculation scheme and the smooth behaviour of the functional dependencies. The access to these data arrays is very fast, which allows performing the vessel maneuvering simulation studies in waves in real-time.

The data for $R_{A W}, U_{d}$, and $N_{d}$ arrays at wavelength $\lambda / L=0.7$, normalized by the square of the wavelength, is illustrated in three-dimensional form in Figs 9, 10, and 11 .

Engineering Sciences, LVIII, 2021, No. 2 


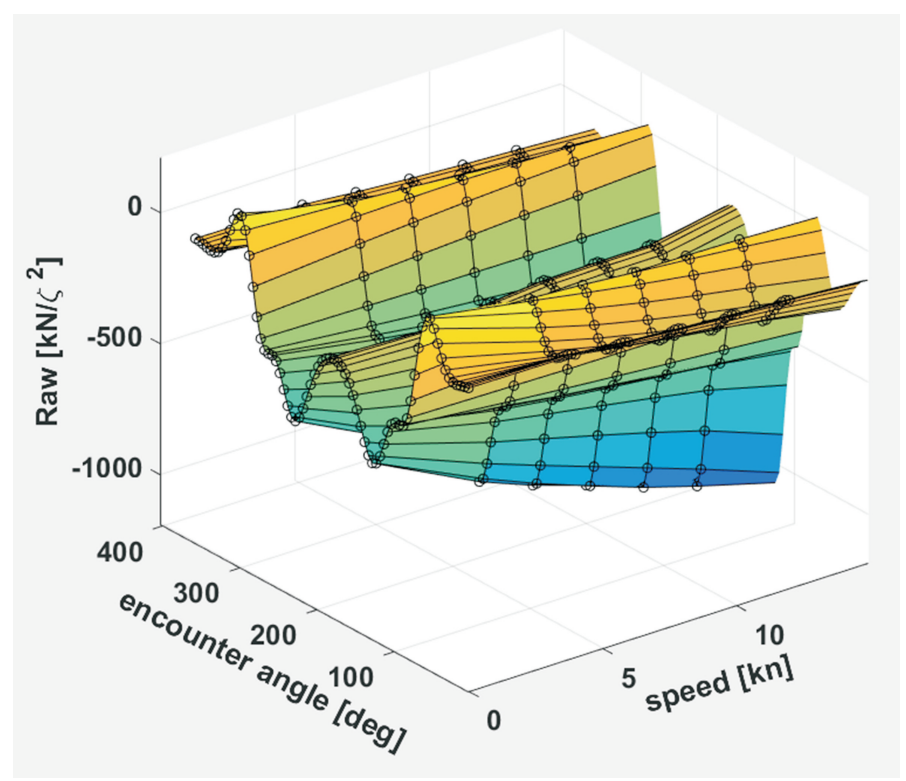

Fig. 9. Added resistance versus $\mu, U$

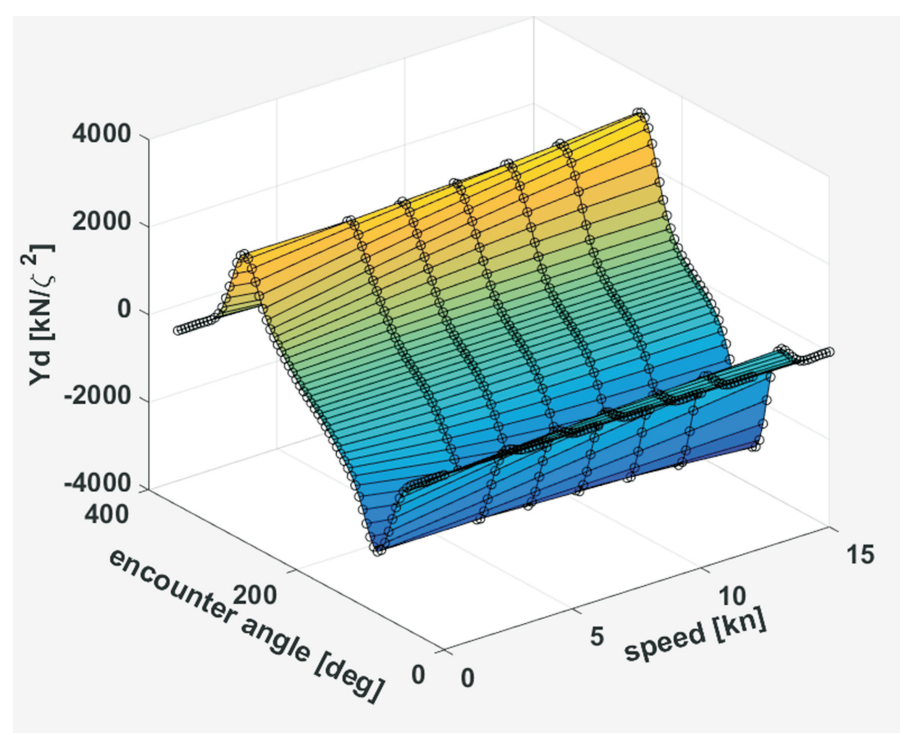

Fig. 10. Lateral drift force versus $\mu, U$ 


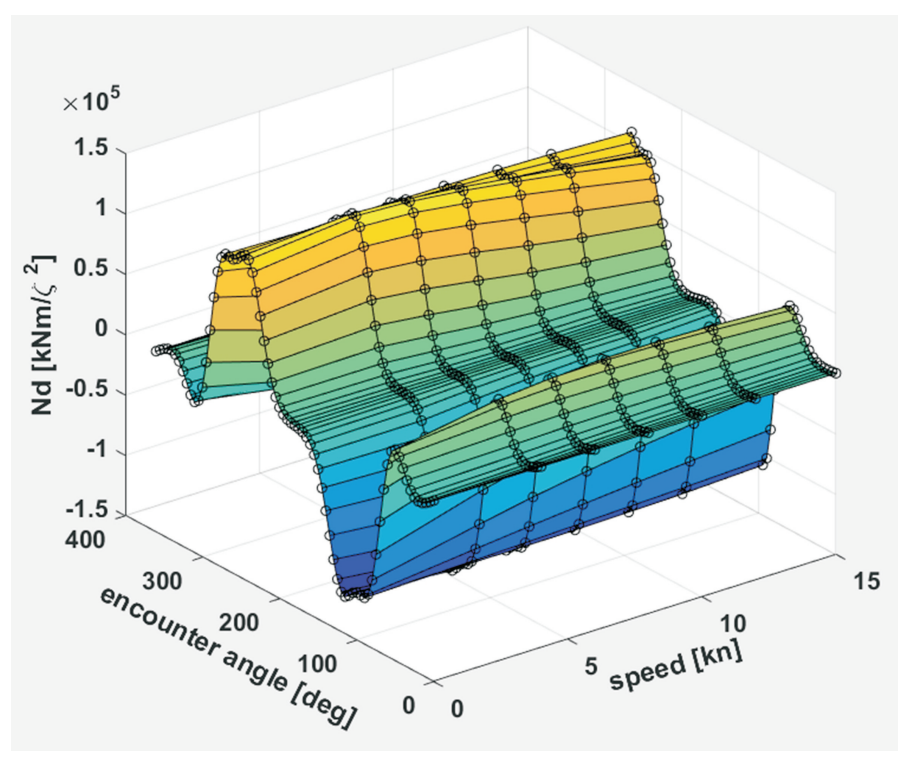

Fig. 11. Yaw drift moment versus $\mu, U$

Table 2. Parameters set of preliminary calculations

\begin{tabular}{|l|c|}
\hline Item & Range of variation \\
\hline Speed $U[\mathrm{kn}]$ & $\langle 0,4,6,8,10,12,15.5\rangle$ \\
\hline Wave direction $\mu[\mathrm{deg}]$ & $\langle 0 \div 360\rangle$ by step of 5 degrees \\
\hline Wavelength $\lambda / L[-]$ & $\langle 0.4 \div 1.5\rangle$ by step of $0.1 ;\langle 2.0\rangle$ \\
\hline Wave steepness $s[-]$ & 0.02 \\
\hline Wave amplitude $\zeta w[\mathrm{~m}]$ & 6.4 \\
\hline
\end{tabular}

\section{SHIP TURNING SIMULATIONS IN REGULAR WAVES}

Based on the obtained data for the wave load and using the results of model tests of KVLCC2 with Planar Motion Mechanism, the simulations of turning maneuver was performed by solving the system of equations (3)-(5). Exemplary results from the calculations compared with the experimental data [13] for wavelengths $\lambda / L=0.7$ and $\lambda / L=1.0$ are shown in Figs 12 and 13, correspondingly.

For analysis of the ship trajectory distortion in waves, two indicators are adopted [13]: distance of the sequential track displacement $H d$ and drifting direction $\mu d$. Because of this, the analysis of the above results shows the following:

- The prediction of drifting distances is approximately; 


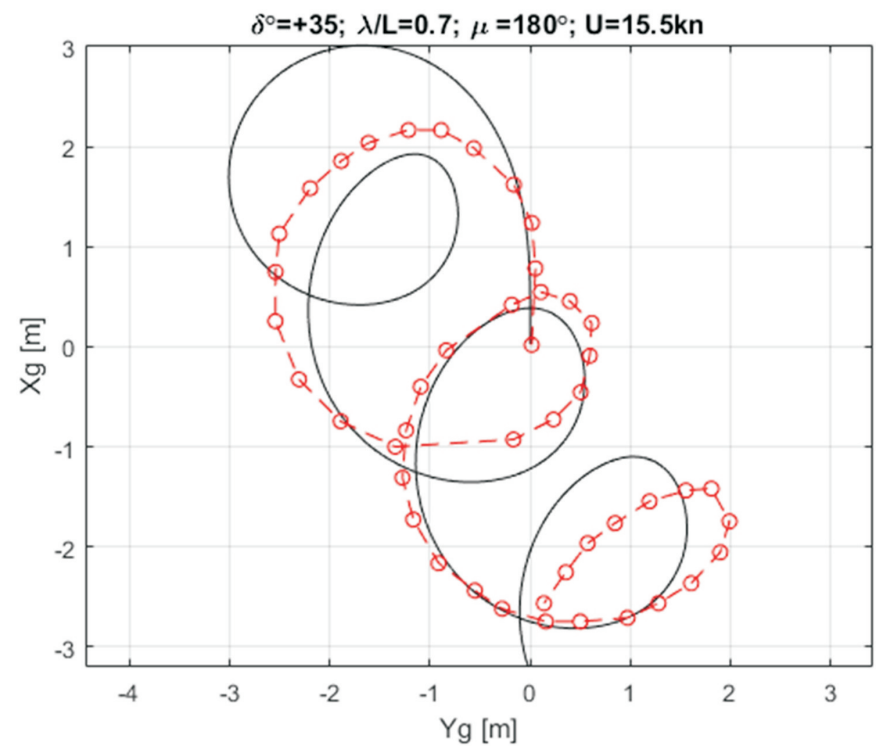

Fig. 12. Turning maneuver in regular waves, $\lambda / L=0.7$

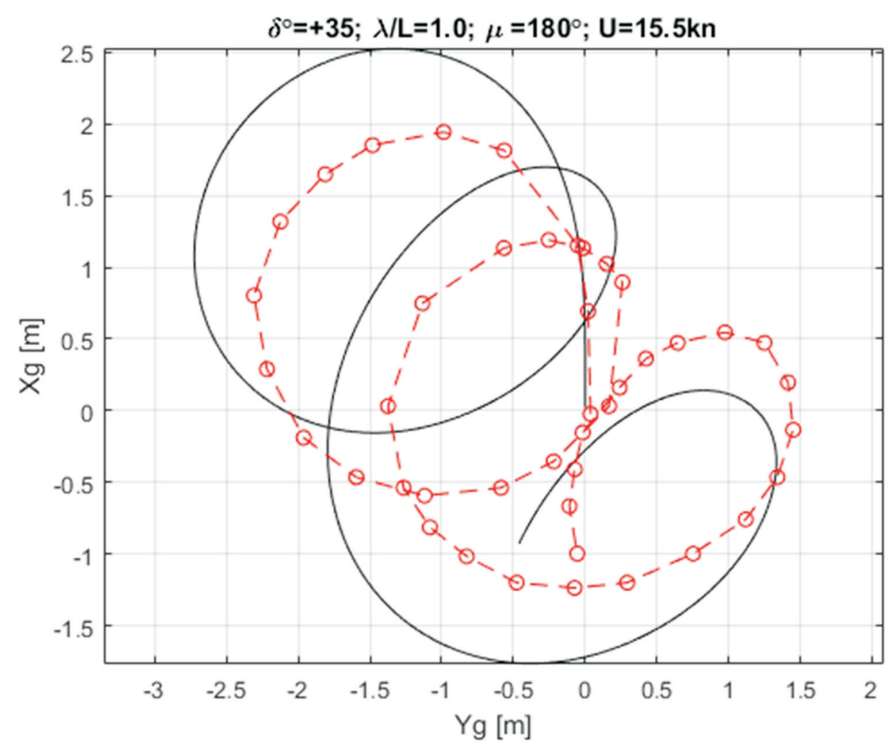

Fig. 13. Turning maneuver in regular waves, $\lambda / L=1.0$ 
- The drifting direction predictions are very close to experimentally measured.

It should be noted that the comparison requires detailed data on some conditions of the conducted model tests, such as a moment of the command to the rudder, characteristics of the waves during successive turnings, etc. By this, first of all, the analysis of the accuracy of wave drift forces and moment predicted by PDSTRIP has to be performed, which is forthcoming in the next stages of the present project.

\section{CONCLUSION}

The following results were achieved in the present study:

- The movements of three ships and the wave forces are predicted and compared with experimental data through potential open-source PDSTRIP. In the first case, the accuracy is very good, while in the second it is satisfactory.

- The procedure for generating a database for ship RAO and wave drift forces in the function of the environmental parameters has been developed.

- The procedure for simulation of ship maneuvers in waves has been applied.

- To improve the predictions, it is planned to use the possibilities of the opensource in the direction: instead of an empirical formula for the added mass $m_{11}$ to apply a more precise solution; to use a more accurate method for estimation of the cross-drag coefficients

The developed procedure can be useful at the ship initial design stage for estimation of vessel maneuverability in waves.

\section{REFERENCES}

[1] SHOPERA EU Project, Energy Efficient Safe SHip OPERAtion (2013-2016), http://shopera.org.

[2] T. Gourlay, A. von Graefe, V. Shigunov, and E. Lataire, Comparison of AQWA, GL RANKINE, MOSE, OCTOPUS, PDSTRIP, and WAMIT with Model Test Results for Cargo Ship Wave-Induced Motions in Shallow Water, in: Proceedings of the OMAE'2015 Conference Canada, 2015, https://doi.org/10.1115/OMAE2015-41691.

[3] G. Parisella and T. Gourlay, Comparison of Open-Source Code NEMOH with WAMIT for Cargo Ship Motions in Shallow Water, Curtin University, Centre for Marine Science and Technology, Tech. Rep. 2016-23, Perth, Australia (2016).

[4] H. Soding And B. Volker, Program PDSTRIP: Public Domain Strip Method (2006), https://sourceforge.net/projects/pdstrip/. 
[5] SIMMAN'2019, International Workshop on Ship Maneuvering, http://www.simman2019.kr/contents/Ship_data_summary.php.

[6] J. Schoop-Zipfel And M. Abdel-Maksoud, A Numerical Model to Determine Ship Maneuvering Motion in Regular Waves, in: Proceedings of MARINE'2011 Conference, Lisbon, Portugal, 2011, http://hdl.handle.net/2117/333243.

[7] ITTC, $15^{\text {th }}$ International Towing Tank Conference, Report of the Seakeeping Committee, the Hague, Netherlands, 1978.

[8] S. Liu and A. Papanikolaou, Estimation of the Maneuvering Characteristics of the DTC Containership Using URANS Based Simulations, Maritime Technology and Engineering 3, Taylor \& Francis Group (2017), ISBN 978-1-139-03000-8.

[9] J. Lee, D. M. Park, And Y. Kim, Comparison of Added Resistance for Different Bow Shapes of KVLCC2, Ship \& Ocean Engineering Research Exchange Meeting, Osaka University, 2013.

[10] H. Yasukawa, Simulation of Ship Maneuvering in Waves $\left(1^{\text {st }}\right.$ report: turning motions), Journal of the Japan Society of Naval Architects and Ocean Engineers (2006) 4127.

[11] A. Papanikolaou, N. Fournakis, D. Chroni, and S. Liu, Simulation of the Maneuvering Behaviour of Ships in Adverse Weather Condition, in: Proceedings of $31^{\text {st }}$ International Symposium on Naval Hydrodynamics, Monterey, USA, 2016.

[12] F. Sprenger, A. Maron, A. Langwinat, and T. Van Zwijnsvoorde, Selected Results of Model Testing, MARINTEK, SHOPERA Project Year 3 Workshop Reports, Lisbon, Portugal, 2015.

[13] D. J. Kim, K. Yun, J. Y. PARK, AND K. Y. Gui, Experimental Investigation on Turning Characteristics of KVLCC2 Tanker in Regular Waves, Journal of Ocean Engineering (2019) 175 197-206, https://doi.org/10.1016/j.oceaneng.2019.02.011.

Received March 25, 2021 